\title{
CENTRAL MASS CONCENTRATION AND BAR DISSOLUTION IN NEARBY SPIRAL GALAXIES

\author{
Mousumi Das, ${ }^{1}$ Peter J. Teuben, ${ }^{1}$ Stuart N. Vogel, ${ }^{1}$ Michael W. Regan, ${ }^{2}$ Kartik Sheth,, 3 \\ ANDREW I. HARRIS, ${ }^{1}$ AND WILliam H. JEFFERYs ${ }^{4}$ \\ Received 2002 July 3; accepted 2002 August 28
}

\begin{abstract}
We use data from the BIMA Survey of Nearby Galaxies (SONG) to investigate the relationship between ellipticity and central mass concentration in barred spirals. Existing simulations predict that bar ellipticity decreases as inflowing mass driven by the bar accumulates in the central regions, ultimately destroying the bar. Using the ratio of the bulge mass to the mass within the bar radius as an estimate of the central mass concentration, we obtain dynamical mass estimates from SONG CO 1-0 rotation curve data. We find an inverse correlation between bar ellipticity and central mass concentration, consistent with simulations of bar dissolution.
\end{abstract}

Subject headings: galaxies: evolution — galaxies: ISM — galaxies: spiral — galaxies: structure

\section{INTRODUCTION}

Bars exert gravitational torques on the gas in the disks of spiral galaxies, resulting in gas inflow toward the center (Quillen et al. 1995; Regan, Vogel, \& Teuben 1997; Regan, Sheth, \& Vogel 1999). This results in a significant increase in the gas mass in the center of a bar (Sakamoto et al. 1999; Sheth 2001), often leading to increased star formation and even starburst activity in the nucleus (Ho, Filippenko, \& Sargent 1997; Jogee, Kenney, \& Smith 1999). Simulations predict that the increased mass concentration may affect the bar structure and even dissolve the bar itself (Kormendy 1982; Hasan \& Norman 1990; Friedli \& Pfenniger 1991; Friedli \& Benz 1993; Hasan, Pfenniger, \& Norman 1993; Norman, Sellwood, \& Hasan 1996). In this paper we investigate whether there is observational evidence for the change in bar shape with mass concentration in the centers of spiral galaxies.

We have used the photometric data of a sample of 13 barred galaxies from the BIMA Survey of Nearby Galaxies (SONG) to determine the bar structure and the CO (1-0) rotation curves to derive central mass concentrations. We use the bar ellipticity $(1-b / a)$, where $a$ is the semimajor axis and $b$ the semiminor axis, as a measure of the bar structure. We define the central mass concentration $f_{\mathrm{mc}}$ as the ratio of the dynamical mass within the bulge to that within the bar radius. The bulge is the most physically distinct region in the galaxy center and easier to measure than other length scales such as core radius, which is used to define central mass concentration in numerical studies (e.g., Norman et al. 1996). We discuss the justification for using the bulge mass in more detail in $\S 5$. To determine $f_{\text {mc }}$, we have used the rotation curves derived from the $\mathrm{CO} J=1-0$ emission in the galaxies. $\mathrm{CO}$ rotation curves were used because $\mathrm{CO}$ traces the kinematics of cold molecular gas, which moves along closed orbits in the plane of a galaxy and hence is a

${ }^{1}$ Department of Astronomy, University of Maryland, College Park, MD 20742; mousumi@astro.umd.edu.

${ }^{2}$ Space Telescope Institute, 3700 San Martin Drive, Baltimore, MD 21218 .

${ }^{3}$ Department of Astronomy, California Institute of Technology, Pasadena, CA 91125.

${ }^{4}$ Department of Astronomy, University of Texas, Austin, TX 78712. good tracer of the dynamical mass distribution in galaxies. In $\S 2$ we describe the galaxy sample and the observational data used in the analysis. In $\S 3$ we discuss how we derived bar ellipticities, and in $\S 4$ we determine $f_{\text {mc }}$ in our sample of galaxies. The statistical analysis is presented in $\S 5$, and we discuss the significance of the results in $\S 6$. We list our conclusions in $\S 7$.

\section{GALAXY SAMPLE}

Our galaxy sample was taken from BIMA SONG; in this survey the centers and inner disks of 44 nearby galaxies were imaged in the CO (1-0) line (Regan et al. 2001; Helfer et al. 2002). The galaxies were observed using the Berkeley-Illinois-Maryland Association (BIMA) array at Hat Creek (Welch et al. 1996). Of the 44 galaxies, 29 are barred and 15 are unbarred. The SONG database includes spatial-velocity cubes that have resolutions of $\sim 4^{\prime \prime}-6^{\prime \prime}$, and $4.1 \mathrm{~km} \mathrm{~s}^{-1}$. More than half of these galaxies had maps that included singledish CO data taken with the NRAO telescope (Helfer et al. 2002). SONG also included a parallel data set of the nearinfrared and optical images of the galaxies. This was important for our study as we needed to determine the bar structure as well as the dynamical mass concentration in our sample.

To determine the central mass concentration, we require in principle only two velocity measurements in a galaxy, i.e., the rotation velocities at the bulge and bar radii. But to be sure that the velocities measured at these radii are not anomalous, we required good velocity coverage for the $\mathrm{CO}$ emission over a significant portion of the inner disk of these galaxies. Thus our sample size was limited by the gas distribution to a subsample of 13 barred galaxies from the BIMA SONG database (Table 1).

\section{BAR SIZE AND ELLIPTICITY}

Bar morphology has an important effect on the gas inflow and star formation in galaxies (Martinet \& Friedli 1997; Aguerri 1999). Bar strength can be quantified either by measuring the bar axis ratio $b / a$ (Martin 1995; Regan \& Elmegreen 1997; Chapelon, Contini, \& Davoust 1999) or alternatively by determining the maximum of the ratio of the tangential force to the mean nonaxisymmetric radial 
TABLE 1

Observed Bar Parameters

\begin{tabular}{|c|c|c|c|c|c|c|c|c|c|}
\hline \multirow[b]{2}{*}{ GaLAXY } & \multirow[b]{2}{*}{$\mathrm{TYPE}^{\mathrm{a}}$} & \multirow[b]{2}{*}{$\begin{array}{c}V_{\text {sys }} \\
\left(\mathrm{km} \mathrm{s}^{-1}\right)\end{array}$} & \multirow[b]{2}{*}{$\begin{array}{l}\text { Distance } \\
(\mathrm{Mpc})\end{array}$} & \multirow[b]{2}{*}{$\begin{array}{l}\text { INCLINATION } \\
\text { (deg) }\end{array}$} & \multicolumn{2}{|c|}{ P.A. } & \multirow[b]{2}{*}{$\begin{array}{l}\text { BAR RADIUS } \\
(\operatorname{arcsec})\end{array}$} & \multirow[b]{2}{*}{$\begin{array}{l}\text { Bulge RADIUS } \\
\text { (arcsec })\end{array}$} & \multirow[b]{2}{*}{$\begin{array}{l}\text { ELLIPTICITY } \\
(1-b / a)\end{array}$} \\
\hline & & & & & $\begin{array}{l}\text { Galaxy } \\
\text { (deg) }\end{array}$ & $\begin{array}{l}\text { Bar } \\
(\mathrm{deg})\end{array}$ & & & \\
\hline NGC $2903 \ldots$ & $\mathrm{SAB}(\mathrm{rs}) \mathrm{bc}$ & 556 & 7.3 & 61.4 & 17 & 24 & 67 & 17 & 0.79 \\
\hline NGC $3184 \ldots$ & $\mathrm{SAB}(\mathrm{rs}) \mathrm{cd}$ & 592 & 8.7 & 21.1 & 180 & 62 & 21 & 13 & 0.21 \\
\hline NGC $3351 \ldots$ & $\mathrm{SB}(\mathrm{r}) \mathrm{b}$ & 774 & 10.1 & 47.5 & 13 & 113 & 54 & 19 & 0.42 \\
\hline NGC $3521 \ldots$ & $\mathrm{SAB}(\mathrm{rs}) \mathrm{bc}$ & 805 & 7.2 & 62.1 & 163 & 162.6 & 25 & 20 & 0.52 \\
\hline NGC $3627 \ldots$ & SAB(s)b & 727 & 11.1 & 62.8 & 173 & 161 & 49 & 26 & 0.68 \\
\hline NGC $3726 \ldots$ & $\mathrm{SAB}(\mathrm{r}) \mathrm{c}$ & 861 & 11.7 & 46.2 & 10 & 32 & 32 & 12 & 0.69 \\
\hline NGC $4303 \ldots$ & $\mathrm{SAB}(\mathrm{rs}) \mathrm{bc}$ & 1562 & 15.2 & 27 & 138 & 2 & 47 & 16 & 0.53 \\
\hline NGC $4321 \ldots$ & $\mathrm{SAB}(\mathrm{s}) \mathrm{bc}$ & 1566 & 16.1 & 31.7 & 153 & 108 & 54 & 20 & 0.51 \\
\hline NGC $4569 \ldots$ & $\mathrm{SAB}(\mathrm{rs}) \mathrm{ab}$ & -221 & 16.8 & 62.8 & 23 & 16 & 65 & 24 & 0.71 \\
\hline NGC $5005 \ldots$ & $\mathrm{SAB}(\mathrm{rs}) \mathrm{bc}$ & 950 & 21.3 & 61.4 & 65 & 74 & 37 & 19 & 0.63 \\
\hline NGC 5248... & $\mathrm{SAB}(\mathrm{rs}) \mathrm{bc}$ & 1158 & 22.7 & 43.6 & 106 & 127 & 80 & 16 & 0.50 \\
\hline NGC 5457... & $\mathrm{SAB}(\mathrm{rs}) \mathrm{cd}$ & 258 & 6.5 & 21.1 & 35 & 80 & 49 & 13 & 0.38 \\
\hline NGC $6946 \ldots$ & $\mathrm{SAB}(\mathrm{rs}) \mathrm{cd}$ & 48 & 6.4 & 31.7 & 64 & 23 & 63 & 15 & 0.44 \\
\hline
\end{tabular}

a From de Vaucouleurs et al. 1995.

force $\left(Q_{b}\right)$ in the bar (Buta \& Block 2001). Recent studies have shown that bar ellipticity is roughly proportional to $Q_{b}$ (Laurikainen, Salo, \& Rautiainen 2002), and so ellipticity appears to be a good measure of the bar strength. In our study we use optical and near-IR images ( $R, I$, and $K$ bands) to determine the bar ellipticity, which we assume provides a reasonable estimate of the bar strength. The $K$ and $I$ bands generally trace the old stellar population in galaxies and thus follow the galactic potential. We used $K$-band images for NGC 3627 (Regan \& Elmegreen 1997) and NGC 6946 (Regan \& Vogel 1995), and I-band images for NGC 2903, NGC 3351, NGC 4303, NGC 4569, NGC 5248, and NGC 5457 . The $R$ band is not as good a tracer of old stars but was all that was available for NGC 3726. For NGC 3184, NGC 3521, NGC 4321 and NGC 5005, we used near-infrared ( $K$ band) images from the 2MASS survey (Jarrett et al. 2000).

The bar was identified from the isophotes in the optical/ IR image. The isophotes were traced using the ellipse task in IRAF, which is based on the photometric technique developed by Jedrzejewski (1987). The bar-defining isophote was assumed to be where the position angle of the elliptical iso-

TABLE 2

Derived Bar Parameters

\begin{tabular}{|c|c|c|c|c|}
\hline \multirow[b]{2}{*}{ GALAXY } & \multicolumn{2}{|c|}{ DyNAMICAL MASS } & \multirow[b]{2}{*}{$\begin{array}{l}\text { Central Mass } \\
\text { Concentration }\end{array}$} & \multirow[b]{2}{*}{$\begin{array}{l}\text { DEPROJECTED } \\
\text { ELLIPTICITY }\end{array}$} \\
\hline & $\begin{array}{c}\text { Bulge } \\
\left(10^{8} M_{\odot}\right)\end{array}$ & $\begin{array}{c}\text { Bar } \\
\left(10^{8} M_{\odot}\right)\end{array}$ & & \\
\hline NGC 2903... & 8 & 170 & 0.05 & 0.59 \\
\hline NGC $3184 \ldots$ & 4 & 10 & 0.45 & 0.24 \\
\hline NGC $3351 \ldots$ & 18 & 306 & 0.06 & 0.60 \\
\hline NGC 3521... & 46 & 69 & 0.67 & 0.04 \\
\hline NGC $3627 \ldots$ & 44 & 245 & 0.18 & 0.43 \\
\hline NGC $3726 \ldots$ & 4 & 61 & 0.07 & 0.62 \\
\hline NGC 4303... & 27 & 218 & 0.12 & 0.53 \\
\hline NGC 4321... & 58 & 374 & 0.16 & 0.52 \\
\hline NGC $4569 \ldots$ & 62 & 448 & 0.14 & 0.42 \\
\hline NGC 5005... & 246 & 760 & 0.32 & 0.33 \\
\hline NGC 5248... & 108 & 596 & 0.18 & 0.40 \\
\hline NGC 5457... & 12 & 77 & 0.15 & 0.38 \\
\hline NGC $6946 \ldots$ & 23 & 173 & 0.13 & 0.45 \\
\hline
\end{tabular}

photes change direction and start tracing the disk of the galaxy (Elmegreen et al. 1996; Laurikainen \& Salo 2000). This isophote was used to determine the position angle and semimajor axis length of the bar. We also determined the intensity profile of the optical or near-IR emission along the bar axis and perpendicular to it, using the IRAF task pvector. All the profiles have a characteristic peak at the center due to the bulge and usually a flat portion that represents the bar. Such profiles have been used in previous studies to determine bar sizes and ellipticities (Elmegreen et al. 1996; Regan \& Elmegreen 1997). We have assumed that the bulge radius is the distance along the bar where the central peak appears to end and the bar profile becomes prominent. In some cases, for example in NGC 4321, there appears to be a double bar since there are two distinct flat portions in the profile (e.g., Knapen et al. 1995). Double bars are evident both in the isophotes and in the intensity profiles. In such cases, we had to carefully examine the profile in order to distinguish the bulge from the rest of the bar.

Bright stars and dust can interfere with the isophote fitting procedure; so to minimize this effect, we fitted the bardefining isophotes using the software suite NEMO (Teuben 1995). To determine deprojected values of the bar parameters, the bar was treated as a two-dimensional ellipse and then projected onto the plane of the galaxy. The parameters involved in the deprojection are the inclination angle of the galaxy and the angle between the bar major axis and galaxy axis. The parameters assumed for the galaxies and the observed bar ellipticities are listed in Table 1 and the deprojected values in Table 2 .

\section{CENTRAL MASS CONCENTRATION FROM ROTATION CURVES}

We have used the rotation curves derived from the $\mathrm{CO}$ (1-0) emission-line observations of BIMA SONG to determine $f_{\mathrm{mc}}$ for our sample of barred galaxies. As noted earlier, the $\mathrm{CO}$ line traces the molecular gas distribution in the bar and should be a good tracer of the bar potential because it is cold, and hence it should settle along closed orbits in the plane of the galaxy. We used the spatial-velocity data cube derived from the spectral line observations to determine the 
zeroth- and first-order moments of the intensity distribution, i.e., the integrated $\mathrm{CO}$ intensity maps and the mean line-of-sight velocity maps for these galaxies.

A detailed description of the methods involved in deriving these rotation curves has been discussed elsewhere (Begeman 1987; P. J. Teuben et al. 2003, in preparation). We summarize the procedure here. We used a two-step process to obtain the $\mathrm{CO}$ velocity fields. First, we generated a mask cube by smoothing the cube at each velocity channel with a $20^{\prime \prime}$ Gaussian; only those pixels with emission brighter than the $3 \sigma$ level in this smoothed cube were allowed by the mask. Then, we further masked the data by including only pixels with brightness above the $2 \sigma$ level in the unsmoothed cube. Moment maps at the full resolution of the data were then generated in the standard way using these two masks. The rotation curves were determined from the velocity fields using the NEMO task rotcur, which is based on the tiltedring model fitting method developed for $\mathrm{H}$ i rotation curves (Begeman 1989). The parameters for the rings are the inclination angle, the position angle, the systemic velocity of the galaxy, and the rotation center of the galaxy. The dynamical center for a galaxy was assumed to be the brightness centroid derived from the $K$-, $I$-, or $R$-band images; this involved examining the intensity contours for the central region and taking the dynamical center to be where the contours converged to a maximum intensity. Both the galaxy center and inclination angle were kept constant for all the rings. An initial estimate of the position angle and the systemic velocity of a galaxy was taken from NED, but it was clear from examination of the rotation curve fits and residuals that the literature values needed to be changed for many galaxies in our sample. We iteratively changed the position angle and the systemic velocity of a galaxy until the errors for the velocity fits in the individual rings in the rotation curve were a minimum. The new values were all close to the literature values. The new improved parameters that we adopted are listed in Table 1. As usual for the tilted-ring method, a rotation velocity is derived for each ring, weighting pixels with respect to the major axis of the galaxy. The width of a ring was set to the mean synthesized beamwidth; for Nyquist sampling, we used rings spaced at half-beam intervals.

The rotational velocities at the bulge radii $\left(R_{\mathrm{blg}}\right)$ and at the bar ends $\left(R_{\mathrm{bar}}\right)$ were interpolated from the rotation curves. We derive the mass concentration as $f_{\mathrm{mc}}=v_{\mathrm{blg}}^{2} R_{\mathrm{blg}} /$ $v_{\mathrm{bar}}^{2} R_{\mathrm{bar}}$, where $v_{\mathrm{blg}}$ and $v_{\mathrm{bar}}$ are the rotational velocities at the bulge radius and at the bar semimajor axis length, respectively. This relation does not include the effects of bulge and disk geometry, which will be different for each galaxy. It also assumes that the magnitude of elliptical streaming is not significant at the bulge radius or bar ends. This is a reasonable assumption in the bulge where the potential is fairly axisymmetric and also at the bar ends where the disk potential begins to be more important than the bar. We discuss evidence supporting this assumption in the next section. The values of $f_{\mathrm{mc}}$ derived for our sample of bars are shown in Table 2. Also shown are the dynamical masses within the bulge and bar of the galaxies.

\section{CORRELATION OF BAR ELLIPTICITY AND CENTRAL MASS CONCENTRATION}

Figure 1 shows the deprojected ellipticities in the plane of the galaxies, plotted against the central mass concentration $f_{\mathrm{mc}}$. The errors for both axes have been calculated using the

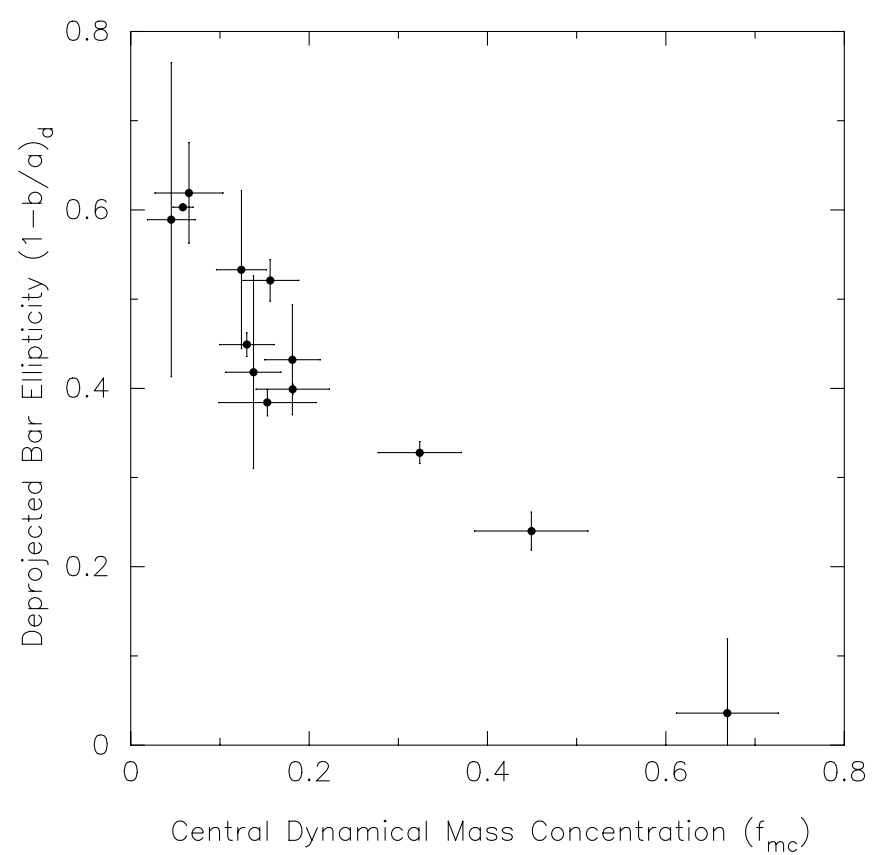

FIG. 1.-Deprojected bar ellipticity $(1-b / a)_{d}$ plotted against the central mass concentration $\left(f_{\mathrm{mc}}\right)$ in the bar. Error bars are $1 \sigma$ for each axis. The three galaxies with the largest central mass concentration, $f_{\mathrm{mc}}$, are in decreasing order NGC 3521, NGC 3184, and NGC 5005, respectively.

standard error propagation equation, based on the uncertainties in the observed quantities (Bevington \& Robinson 1992). The error along the ellipticity axis includes a coefficient due to the deprojection of the bar onto the plane of the galaxy. It is clear even from just visual inspection that there is a correlation between bar ellipticities and $f_{\mathrm{mc}}$ in the galaxies. However, for a more quantitative estimate of the correlation, we have determined a linear correlation coefficient for the sample using two different methods.

An accurate estimate of the correlation coefficient should include the errors on both axes, which leads to a weighted correlation coefficient, but this is difficult to obtain in practice (Feigelson \& Babu 1992). So we used a simple Monte Carlo simulation to determine a mean weighted correlation coefficient. We generated 20,000 linear fits to the points in the sample where each line randomly sampled the errors on both axes. The mean correlation coefficient $\bar{r}$ of all the fits was assumed to be the weighted correlation coefficient for the sample. We obtained a value of $\bar{r}=-0.86$, which indicates a significant correlation. It is also important to determine the $p$-value $P_{r}$ corresponding to such a linear correlation coefficient (Bevington \& Robinson 1992). For $\bar{r}=-0.86, P_{r}<0.001$, so the probability that they are from a random sample is small. The second approach was to use the Bayesian model fitting method (Sivia 1996; Loredo 1990). This technique determines the probability of a correlation assuming the errors on both axes to be independent and Gaussian. The resulting integral was solved using the Markov chain Monte Carlo technique (Casella \& George 1992). This more rigorous method, which used conventional priors for the analysis, resulted in a mean correlation coefficient of $\bar{r}=-0.75 \pm 0.1$ and the posterior probability of the uncorrelated model is 0.02 . Thus both methods confirm the significant correlation evident by eye.

From Figure 1 it is clear that the three galaxies NGC 5005, NGC 3184, and NGC 3521, which have ellipticity less 
than 0.35 , are important for the correlation. So we have examined these galaxies in closer detail and compared their bar properties and rotation curves with other observations in the literature.

$N G C 5005$. - In the $K$ band, this galaxy appears to have a strong bulge and a fairly round bar. But there are two features that at first glance appear inconsistent with this. First, there are two straight structures parallel to the length of the bar; they make the bar appear more elliptical than our estimate from isophote fitting. On closer inspection they appear offset from one another, rather like dust lanes. However, dust emission should not be prominent in the $K$ band, so these structures cannot represent dust lanes; their origin is thus not clear and requires further investigation. An alternative estimate of how round a bar is can be obtained from the bar strength parameter $Q_{b}$. E. Laurikainen (2002, private communication) found a low value of $Q_{b}=0.16$ for the bar strength in this galaxy. The second feature is the velocity field, which exhibits prominent shocks that are typically considered a feature of strong bars, not weaker bars such as NGC 5005. However, using the Piner, Stone, \& Teuben (1995) hydrodynamical code, we simulated a galaxy with a bar axis ratio similar to NGC 5005 and found strong shocks similar to that observed. Thus we conclude that despite the strong shocks, a weak bar with $Q_{b}=0.16 \pm 0.5$ is possible.

The rotation curve of this galaxy is affected by beam smearing, which is made worse by the high inclination angle of this galaxy (61.4). Also, since the bar is fairly closely aligned with the major axis in this galaxy, the positionvelocity (PV) plot is affected by the elliptical streaming of gas in the bar. This explains why the rotation curve has a velocity at the bulge radius lower than that seen in the PV diagram; this was also evident when we compared our rotation curve with the PV plot of Sakamoto, Baker, \& Scoville (2000). However, the beam smearing effect is important for mainly the inner 2-3 beamwidths, which is well within the bulge radius for this galaxy. Also, the effect of elliptical streaming is considerably reduced in deriving the rotation curve because velocities are azimuthally averaged over annuli. We thus believe our isophote measurement of the bar ellipticity and the rotation curve determination of $f_{\mathrm{mc}}$ are both reasonable estimates for NGC 5005.

NGC 3184. - This galaxy has both a large bulge and a round bar. The bar is not prominent in the $K$-band image. However the $\mathrm{CO}$ distribution shows the classic response of gas in a barred potential, with trailing spiral arms emerging from the ends of the bar. The bulge is a little over half the bar size and is very bright in the near-IR, which may indicate a large mass concentration in the center of the galaxy.

$N G C 3521$. - The ellipticity of this galaxy is so low that it might be questionable whether it is in fact a barred galaxy. But first we note that this galaxy is classified as a barred galaxy in the RC3 catalog (Table 1). However, the bar is not easy to distinguish. This is partly because of the low ellipticity of the bar but also because of the high inclination of the galaxy $\left(62^{\circ}\right)$. We found evidence for the bar in the $K$-band photometry and also in the intensity profile along the major axis of the galaxy. Also, Zeilinger et al. (2001) find evidence for the bar both in their $R$-band photometry and in the stellar kinematics derived from the long-slit spectra obtained along different axes in the galaxy. This leads us to believe that there is a rather round bar in this galaxy. The bulge is very bright and very large; it is over three-fourths the size of the bar, and this indicates that it may also be very massive.
It therefore appears that the three galaxies with low measured ellipticities are indeed barred galaxies with relatively low ellipticity and with high central mass concentrations. Nonetheless, it will be important to confirm the trend identified here using a larger sample of galaxies.

The last issue that should be discussed is the effect of elliptical streaming in the bar, due to gas moving on $x_{1}$ and $x_{2}$ orbits. The $x_{1}$ orbits are aligned along the bar within corotation radius, and the $x_{2}$ orbits are elongated perpendicular to the length of the bar (Contopoulos \& Papayannopoulos 1980; Binney \& Tremaine 1987). We have tried to minimize the effect of elliptical streaming by measuring velocities at the bulge and bar radii. We assume that we are measuring the $x_{2}$ orbits at the edge of the bulge and the $x_{1}$ orbits at the edge of the bar. For a bar aligned with the major axis of the galaxy, $v_{\text {blg }}$ is measured at the pericenter of its $x_{2}$ orbit and is therefore larger than a circular orbit consistent with the mass interior to that radius. Equally so, $v_{\text {bar }}$ is measured at the apocenter of its $x_{1}$ orbit and is therefore smaller then the corresponding circular orbit. Therefore $f_{\mathrm{mc}}$ would be overestimated from a value consistent with the mass distribution. Conversely, for bars aligned along the minor axis, $f_{\mathrm{mc}}$ would be underestimated. We did not see such a trend in Figure 1. We should also note that the rotation curve is derived from a two-dimensional velocity field which will tend to average out this effect.

\section{DISCUSSION}

Figure 1 shows that galaxies which have more mass concentrated in their bulges have rounder bars. However, the existence of a correlation does not necessarily imply a causal relationship. For example, it might be that some other parameter used to calculate the masses is more relevant. In particular, the central mass concentration is calculated as $f_{\mathrm{mc}}=v_{\mathrm{blg}}^{2} R_{\mathrm{blg}} / v_{\mathrm{bar}}^{2} R_{\mathrm{bar}}$; it might be that the relative bulge size $l_{c}=R_{\mathrm{blg}} / R_{\mathrm{bar}}$ is more relevant than the dynamical mass concentration. To investigate this, we plotted ellipticities against $l_{c}$ for 25 barred SONG galaxies (Fig. 2). Comparison with Figure 1 shows that the correlation of ellipticity with $l_{c}$ is not as good as with $f_{\text {mc }}$; the linear correlation coefficient (unweighted) is -0.56 , significantly less than for $f_{\mathrm{mc}}$. We therefore conclude that $f_{\mathrm{mc}}$ is more likely than $l_{c}$ to be relevant for the central mass concentration. The points in Figure 2 are coded to represent the three Hubble types, i.e., early-, intermediate- and late-type barred galaxies. Early type galaxies have large, bright bulges, whereas late-type galaxies appear to have less prominent bulges (Hubble 1926). In Figure 2 the different types are dispersed over the whole plot; there does not appear to be any correlation of bar ellipticity with Hubble type. This may because the Hubble sequence is based on the optical brightness of bulges, which may not always be a good measure of the bulge mass. We thus conclude that bar ellipticity is less correlated with bulge size or Hubble type than with central mass concentration. Thus Figure 1 indicates that bar ellipticity, which approximately measures bar strength, decreases with mass concentration in the bar.

The correlation is perhaps not surprising, since a spherical mass concentration in the center of a bar will tend to decrease the nonaxisymmetric effect of the bar. Nonetheless, it is worth pointing out that this correlation is predicted by secular evolution models, as we now discuss. Simulations show that bars drive gas inward, resulting in central star 


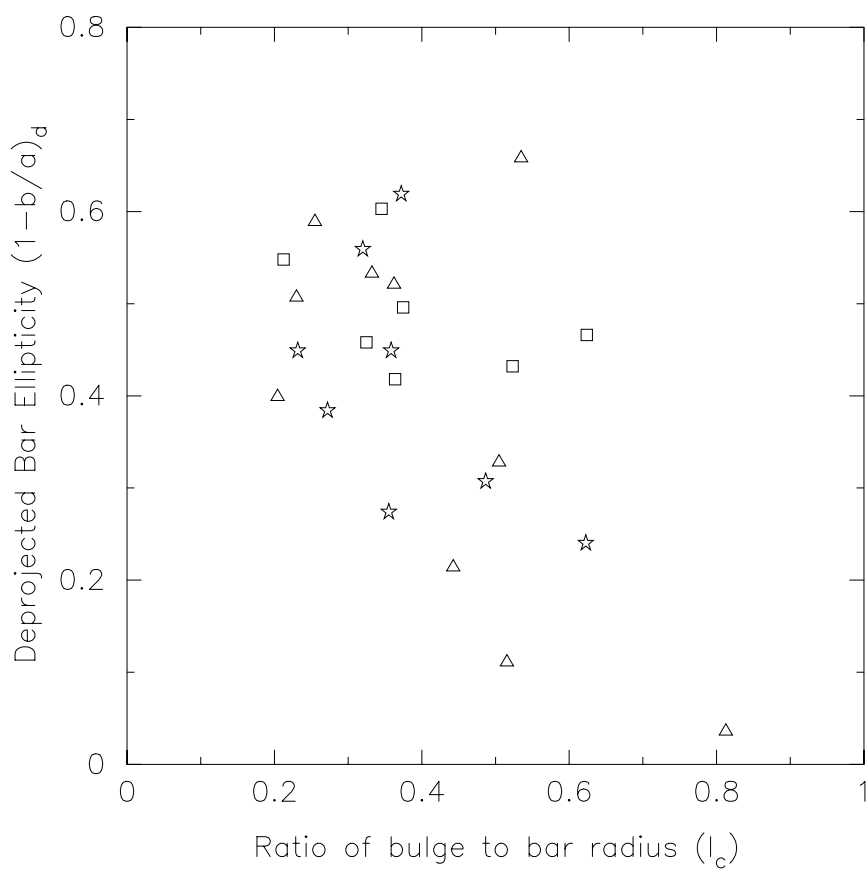

FIG. 2.-Deprojected bar ellipticity plotted against the ratio of bulge to bar radii $\left(l_{c}\right)$ for $25 \mathrm{SONG}$ galaxies. The open squares represent early-type galaxies, the open triangles represent intermediate galaxies, and the stars represent late-type galaxies.

formation and a consequent buildup of the central mass. The central mass concentration affects the stability of the bar supporting $x_{1}$ orbits so that the bar finally dissolves leading to the transformation of a barred spiral to an unbarred one (Friedli \& Benz 1993). Other simulations indicate that it may be the massive core that affects the $x_{1}$ orbits, causing them to become stochastic and leading to the dissolution of the bar (Hasan et al. 1993; Norman et al. 1996; Hozumi \& Hernquist 2001). These models also explain the lack of bars with low ellipticity because once $f_{\text {mc }}$ reaches a certain range, the bar evolves very rapidly. It has also been suggested that bar dissolution may be due to the scattering of stars by a large mass concentration in the center of a galaxy (Norman, May, \& van Albada 1985; Gerhard \& Binney 1985) or even a prolate halo (Ideta \& Hozumi 2000).

We have, however, measured the bulge concentration $f_{\mathrm{mc}}$ and not the core mass. Nonetheless, $f_{\text {mc }}$ may be a useful measure of the core mass concentration. Observations of the black hole or core masses in galaxies indicate they are well correlated with the bulge mass (Magorrian et al. 1998; Richstone et al. 1998; Ferrarese \& Merritt 2000; Gebhardt et al. 2001). This means that though $f_{\mathrm{mc}}$ does not directly measure a nuclear mass concentration, it may be a reasonable measure of the effect of a massive core on the overall bar morphology. Table 2 shows the dynamical masses in the bulge and bar calculated using the approximation, $M_{\text {dyn }} \sim r v^{2} / G$. Assuming that galaxies have a dynamical mass lying in the range $10^{11}-10^{12} M_{\odot}$, some of the galaxies in our sample have bulge masses that are a few percent of the total galaxy mass. This may be large enough to affect the $x_{1}$ orbits in these galaxies, and further increase could lead to bar dissolution, which will leave behind a spheroidal bulge in the center of the galaxy. This is but one of several processes such as accretion or bending instabilities that lead to bulge formation (e.g., Raha et al. 1991; Carlberg 1992). This should not, however, prevent galaxies from reforming bars again if the disk is cool enough; this must evidently be the case since a significant fraction of all spiral galaxies are unbarred. Thus bar formation, dissolution, and bulge formation may be an ongoing evolutionary process in galaxies (Bournaud \& Combes 2002).

We end the discussion on a note of caution in using Figure 1 in support of bar dissolution models. First, a larger sample is required to confirm the apparent correlation. Second, even though ellipticity and central mass concentration are correlated, this of course does not require a causal connection between the two. Third, if bars dissolve significantly faster than a Hubble time, we need to understand how they reform since there is a significant fraction of barred galaxies.

\section{CONCLUSIONS}

We have used the BIMA SONG survey data to determine ellipticities and mass concentrations in the centers of nearby barred galaxies. We have used optical or near-infrared images to determine bar shapes and the $\mathrm{CO}(1-0)$ rotation curves to derive dynamical masses in the bulge and bar regions of the galaxies. (1) We find an apparent correlation between the bar ellipticity and the central mass concentration. For our sample of 13 galaxies a conservative analysis yields a correlation coefficient of $\sim-0.8$. The probability that the parent sample is uncorrelated is 0.012 , which indicates that it is a statistically significant correlation. (2) The correlation suggests that bar structure is affected by the dynamical mass concentration in the bulge. This may provide evidence that bars evolve as gas flows inward and mass accumulates in their centers, indicating that the mass concentration affects the bar structure and may eventually dissolve the bar.

M. D. thanks S. Jogee, E. Laurikainen, W. W. Zeilinger, and K. Sakamoto for useful discussions; we thank the SONG team for providing the entire data set used in this paper. This work is partially supported by NSF AST 9981289. This research has made use of the NASA/ IPAC Infrared Science Archive, which is operated by the Jet Propulsion Laboratory, California Institute of Technology, under contract with the National Aeronautics and Space Administration.

\section{REFERENCES}

Aguerri, J. A. L. 1999, A\&A, 351, 43

Begeman, K. G. 1987, Ph.D. thesis, Univ. Groningen 1989, A\&A, 223, 47

Bevington, P. R., \& Robinson, D. K. 1992, Data Reduction and Error Analysis for the Physical Sciences (2d ed.; New York: McGraw-Hill)

Binney, J., \& Tremaine, S. 1987, Galactic Dynamics (Princeton: Princeton Univ. Press)

Bournaud, F., \& Combes, F. 2002, A\&A, 392, 83

Buta, R., \& Block, D. L. 2001, ApJ, 550, 243
Carlberg, R. G. 1999, in The Formation of Galactic Bulges, ed. C. M. Carollo, H. C. Ferguson, \& R. F. G. Wyse (Cambridge: Cambridge Univ. Press), 64

Casella, G., \& George, E. I. 1992, American Statistician, 46, 167

Chapelon, S., Contini, T., \& Davoust, E. 1999, A\&A, 345, 81 Contopoulos, G., \& Papayannopoulos, T. 1980, A\&A, 92, 33

de Vaucouleurs, G., de Vaucouleurs, A., Corwin, H. G., Buta, R. J. Paturel, G., Fouqué, P. 1995, Third Reference Catalogue of Bright Galaxies (New York: Springer) (RC3) 
Elmegreen, B. G., Elmegreen D. M., Chromey, F. R., Hasselbacher, D. A., \& Bissel, B. A. 1996, AJ, 111, 2233

Feigelson, E. D., \& Babu, G. J. 1992, ApJ, 397, 55

Ferrarese, L. \& Merritt, D. 2000, ApJ, 539, L9

Friedli, D., \& Benz, W. 1993, A\&A, 268, 65

Friedli, D., \& Pfenniger, D. 1991, in IAU Symp. 146, Dynamics of Galaxies and Their Molecular Cloud Distributions, ed F. Combes \& F. Casoli (Dordrecht: Reidel), 362

Gebhardt, K., et al. 2000, ApJ, 539, L13

Gerhard, O. E., \& Binney, J. 1985, MNRAS, 216, 467

Hasan, H., \& Norman, C. 1990, ApJ, 361, 69

Hasan, H., Pfenniger, D., \& Norman, C. 1993, ApJ, 409, 91

Helfer, T. T. 2002, ApJS, submitted

Ho, L. C., Filippenko, A. V., \& Sargent, W. L. W. 1997, ApJ, 487, 591

Hozumi, S., \& Hernquist, L. 2002, ApJL, submitted

Hubble, E. 1926, ApJ, 64, 321

Ideta, M., \& Hozumi, S. 2000, ApJ, 535, L91

Jarrett, T. H., Chester, T., Cutri, R., Schneider, S., Skrutskie, M., \& Huchra, J. P. 2000, AJ, 119, 2498

Jedrzejewski, R. I. 1987, MNRAS, 226, 747

Jogee, S., Kenney, J. D. P., \& Smith, B. J. 1999, ApJ, 526, 665

Knapen, J. H., Beckman, J. E., Shlosman, I., Peletier, R. F., Heller, C. H., \& de Jong, R. S. 1995, ApJ, 443, L73

Kormendy, J. 1982, in Morphology and Dynamics of Galaxies, ed. L. Martinet \& M. Mayor (Sauverny: Geneva Obs.), 113

Laurikainen, E., \& Salo, H. 2000, A\&AS, 141, 103

Laurikainen, E., Salo, H., \& Rautiainen, P. 2002, MNRAS, 331, 880

Loredo, T. J. 1990, Maximum Entropy and Bayesian Methods, ed. P. F. Fougere (Dordrecht: Kluwer), 81
Magorrian, J. et al. 1998, AJ, 115, 2285

Martin, P. 1995, AJ, 109, 2428

Martinet, L., \& Friedli, D. 1997, A\&A, 323, 363

Norman, C., May, A., \& van Albada, T. 1985, ApJ, 296, 20

Norman, C., Sellwood, J. A., \& Hasan, H. 1996, ApJ, 462, 114

Piner, B. G., Stone, J. M., \& Teuben, P. J. 1995, ApJ, 449, 508

Quillen, A. C., Frogel, J. A., Kenney, J. D. P., Pogge, R. W., \& Depoy, D. L. 1995, ApJ, 441, 549

Raha, N., Sellwood, J. A., James, R. A., \& Kahn, F. D. 1991, Nature, 352, 411

Regan, M. W., \& Elmegreen, D. B. 1997, AJ, 114, 965

Regan, M. W., Sheth, K., \& Vogel, S. 1999, ApJ, 526, 97

Regan, M. W., \& Vogel, S. N. 1995, ApJ, 452, L21

Regan, M. W., Vogel, S. N., \& Teuben, P. J. 1997, ApJ, 482, L143

Regan, M. W., et al. 2001, ApJ, 561, 218

Richstone, D., et al. 1998, Nature, 395, A14

Sakamoto, K., Baker, A. J., \& Scoville, N. Z. 2000, ApJ, 533, 149

Sakamoto, K., Okumura, S. K., Ishizuki, S., \& Scoville, N. Z. 1999, ApJ, 525,691

Sheth, K. 2001, Ph.D. thesis, Univ. Maryland

Sivia, D. S. 1996, Data Analysis: A Bayesian Tutorial (New York: Oxford Univ. Press)

Teuben, P. J. 1995, in ASP Conf. Ser. 77, Astronomical Data and Software Systems IV, ed. R. A. Shaw, H. E. Payne, \& J. J. E. Hayes (San Francisco: ASP), 398

Welch, W. J., et al. 1996, PASP, 108, 93

Zeilinger, W. W., Vega Beltran, J. C., Rozas, M., Beckman, J. E., Pizella, A., Corsini, E. M., \& Bertola, F. 2001, Ap\&SS, 276, 643 